\title{
Epstein-Barr Virus-Associated Acute Liver Failure Present in a 67-Year-Old Immunocompetent Female
}

\author{
Wei Zhang ${ }^{\mathrm{a}, \mathrm{b}}$, Betty Chen ${ }^{\mathrm{a}}$, Yongxin Chen ${ }^{\mathrm{a}}$, Robin Chamberland ${ }^{\mathrm{a}}$, Alexa Fider-Whyte ${ }^{\mathrm{b}}$, Julia Craig ${ }^{\mathrm{c}}$, \\ Chintalapati Varma ${ }^{\mathrm{c}}$, Alex S. Befeler ${ }^{\mathrm{b}}$, Adrian M. Di Bisceglie ${ }^{\mathrm{b}}$, Peter Horton ${ }^{\mathrm{c}}$, \\ Jin-Ping Lai ${ }^{\mathrm{a}, \mathrm{d}}$
}

\begin{abstract}
Acute liver failure (ALF) is a rare illness with a high mortality rate. The only favorable management is emergent liver transplantation. About $13 \%$ of ALF cases have no clear etiology. Epstein-Barr virus (EBV)-associated ALF accounts for less than $1 \%$ of all ALF cases, and is seen mostly in adults younger than 40 years. There are only a few cases of EBV-associated ALF in elderly immunocompromised adults. We report a case of ALF in an immunocompetent 67-year-old woman caused by EBV infection that was treated by orthotopic liver transplantation (OLT). The diagnosis of EBV-associated ALF was established by EBV-DNA polymerase chain reaction (PCR) and EBVencoded RNA (EBER-RNA) in situ hybridization (EBER-RISH). The patient is currently doing well 6 months after transplantation without any evidence of clinical EBV infection. This case illustrates the importance of early recognition and diagnosis of EBV-associated ALF by detection of EBV from liver biopsy, especially when patients are immunocompetent and other causes are excluded. To the best of our knowledge, this is the first case of EBV-associated ALF present in an immunocompetent elderly female.
\end{abstract}

Keywords: Epstein-Barr virus; Acute liver failure; Liver biopsy

\section{Introduction}

Acute liver failure (ALF) is a rare but life-threatening illness which occurs mostly in young adults without any known underlying liver disease $[1,2]$. In the United States, the most

Manuscript accepted for publication September 09, 2016

aDepartment of Pathology, Saint Louis University School of Medicine, St Louis, MO 63104, USA

bepartment of Internal Medicine, Saint Louis University School of Medicine, St Louis, MO 63104, USA

'Department of Surgery, Saint Louis University School of Medicine, St Louis, MO 63104, USA

${ }^{\mathrm{d} C}$ Corresponding Author: Jin-Ping Lai, Department of Pathology, Saint Louis University School of Medicine, 1402 South Grand Blvd, St. Louis, MO 63104, USA. Email: jinpinglai@slu.edu

doi: http://dx.doi.org/10.14740/gr718e common cause of ALF is acetaminophen overdose (39\%) but in $13 \%$ of cases, the causes are indeterminate [3]. Epstein-Barr virus (EBV) is a DNA virus associated with infectious mononucleosis (IM) in children and young adults. It may cause moderate and transitory increase of liver enzymes; however, in rare cases, severe liver injury and even fatal ALF may occur [4]. The only definitive treatment for EBV-associated ALF is liver transplantation [5]. We report a case of successful orthotopic liver transplantation (OLT) in a 67-year-old female who presented with EBV-associated ALF.

\section{Case Report}

A 67-year-old woman with bilateral osteoarthritis of the knee, hypothyroidism and recurrent deep vein thrombosis presented with 3 weeks of fatigue and a 1-week history of jaundice. Her medications include diclofenac, rivaroxaban, desiccated thyroid, alprazolam, selenium and glucosamine. She denied any illicit drug use, alcohol use or recent travel. Four weeks prior to admission, the patient was found to have mildly elevated serum aminotransferases during a routine visit. Simvastatin was discontinued; however, her aminotransferases continued to be increased. Three weeks prior to admission, patient began to feel tired. One week prior to admission, she reported to become jaundiced and her aminotransferases were found to be elevated to about $1,000 \mathrm{U} / \mathrm{L}$, with an INR of 2.7. A liver biopsy was performed at an outside institution. A diagnosis of massive hepatic necrosis of uncertain etiology was made. One day prior to admission, her serum alanine aminotransferase (ALT) was 3,542 U/L, aspartate aminotransferase (AST) was 3,610 U/L, alkaline phosphatase (ALP) was $266 \mathrm{U} / \mathrm{L}$ and her INR was 2.7 .

On admission, her vital signs were normal, and a physical exam was positive only for jaundice. She did not have abdominal distention, tenderness, hepatomegaly or splenomegaly. Laboratory findings included a white blood cell count of $13,200 / \mu \mathrm{L}$ (neutrophils $84.9 \%$, lymphocytes $12.4 \%$, monocytes $3.5 \%$ and eosinophils $0.2 \%$ ). Hemoglobin was $15.0 \mathrm{~g} / \mathrm{dL}$ and a platelet count was $195,000 / \mu \mathrm{L}$. Her serum electrolytes were normal. Serum creatinine was $3.8 \mathrm{mg} / \mathrm{dL}$. Her ALT was $2,193 \mathrm{U} / \mathrm{L}$, AST was $1,236 \mathrm{U} / \mathrm{L}$, ALP was $215 \mathrm{U} / \mathrm{L}$ and INR was 2.9.

Abdominal ultrasonography showed an edematous liver 

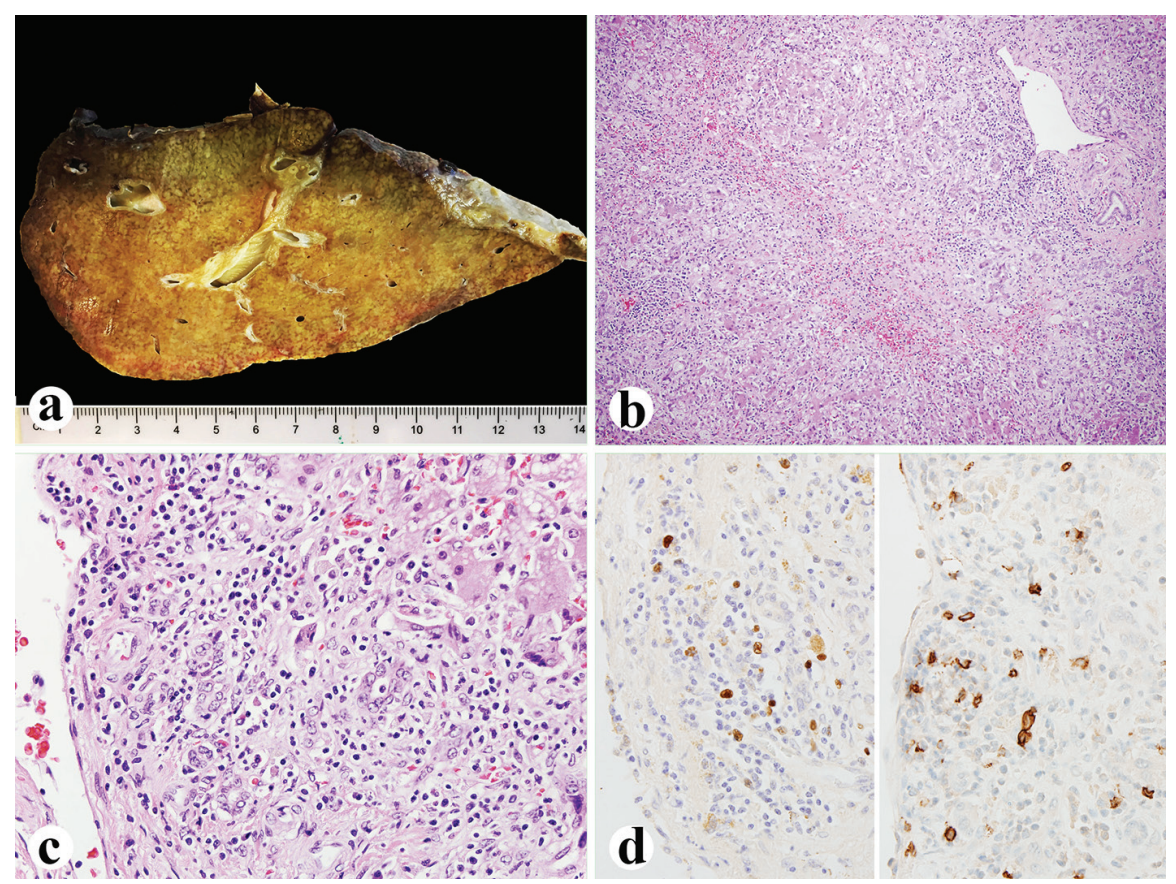

Figure 1. Explanted liver. (a) Small explanted liver weighing 720 g. (b, c) Histologically, the liver showing $75 \%$ of parenchyma extinction and massive necrosis with sinusoidal lymphocytosis and atypical lymphocytes (H\&E, b: $\times 100 ; \mathrm{c}: \times 400)$. (d) EBV in situ hybridization (left, $\times 600$ ) and CD20 immunostain (right, $\times 600$ ) showing numerous EBV-positive atypical B lymphocytes.

with ascites. There was no splenomegaly. The gallbladder wall was markedly thickened. Hepatic artery, hepatic veins, and portal vein were patent, with a normal direction of flow by Doppler study.

Serum acetaminophen level was $7 \mu \mathrm{g} / \mathrm{mL}$. HAV antibody, HBsAg, HCV and HEV antibody were all negative. Serum IgG and IgM antibodies to HSV were slightly elevated. Blood tests for human immunodeficiency virus (HIV, HIV1/2 antibody and p24 antigen), cytomegalovirus (CMV), rubella and rubeola were negative. Serum alpha1-antitrypsin and ceruloplasmin were normal. Antinuclear antibody, anti-mitochondrial antibody and anti-smooth muscle antibody were negative.

The patient was initially diagnosed with ALF possibly, secondary to diclofenac use, because she had begun to take diclofenac potassium $50 \mathrm{mg}$ twice daily since 5 months ago. She was admitted to the ICU and received multiple transfusions of fresh-frozen plasma (FFP) to correct her coagulopathy. She was evaluated and was listed for liver transplantation. She was given midodrine and octreotide and underwent hemodialysis for hepatorenal syndrome. Despite multiple transfusions of FFP, her coagulopathy worsened, and her INR was 3.7 the following day. Her serum ALT and AST decreased rapidly in the following 2 days and the patient became more lethargic and somnolent, and difficult to arouse. Four days after admission, she underwent a successful liver transplantation from a CMV-negative, EBV (IgG) positive cadaveric donor. The explanted liver was significantly smaller than normal indicating acute failure of the liver (Fig. 1a). After transplantation, she received thymoglobulin, methylprednisolone taper and was later switched to prednisone taper, and she also started tacrolimus 4 days after transplantation.
Her histological report of the explanted liver showed that there was $75 \%$ massive hepatic parenchyma necrosis (Fig. 1b). There were also acidophilic bodies, microvesicular steatosis and mild macrovesicular steatosis present in the residual hepatic parenchyma. There was a mixed population of inflammatory cells with predominant lymphocytes with sinusoidal lymphocytosis (Fig. 1c). Numerous atypical B lymphocytes were present in the collapsed parenchyma and sinusoids. They were positive for EBV RNA by in situ hybridization (Fig. 1d, left) and positive for CD20 immunostain (1:100 dilution, horse radish peroxidase technique; Dako, Carpenteria, CA) (Fig. $1 \mathrm{~d}$, right). HSV staining of the liver tissue was negative although the serum antibodies were slightly higher than normal. A review of the liver biopsy 5 days prior to admission showed about $25 \%$ hepatic parenchyma necrosis with bridging necrosis (Fig. 2a, b), with positive EBV in situ hybridization (Fig. 2c). A whole blood assay for EBV DNA using PCR of EDTA anticoagulated peripheral blood showed 462,000 copies/mL. She was started on intravenous acyclovir immediately after the diagnosis of EBV infection, and later she was switched to oral acyclovir which was administered for 3 months. Serum ALP after transplantation was initially increased but decreased immediately (Fig. 2d) after starting intravenous acyclovir. Two weeks after transplantation, EBV viral capsid antigen (VCA)IgG test was positive and VCA-IgM was negative, EBV D early antigen (EA-D) and Epstein-Barr nuclear antigen (EBNA) were negative. One month later, the transplanted liver underwent biopsy and showed negative CD20 and EBV RNA (data not shown). Repeat EBV DNA PCR on blood was negative. The patient is presently doing well 6 months after liver transplantation with no evidence of clinical EBV infection or lym- 

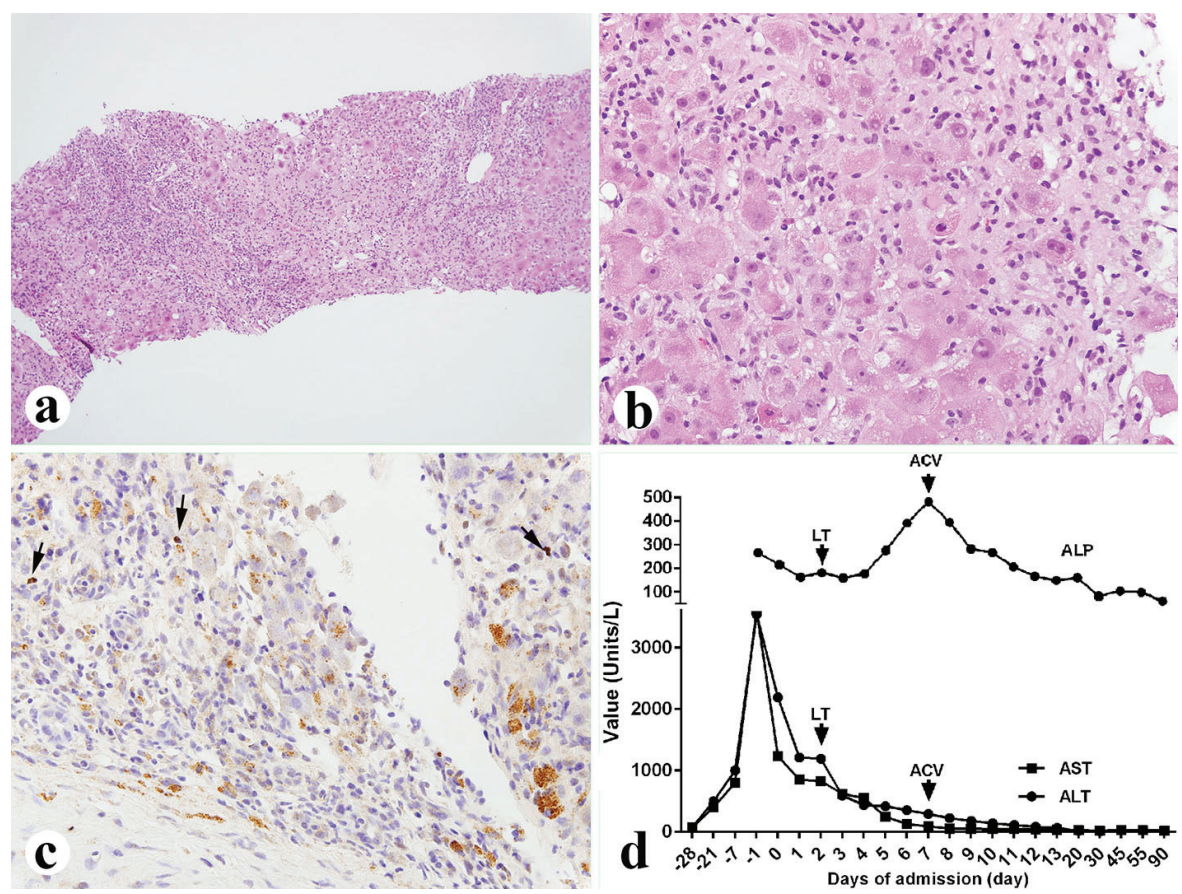

Figure 2. Liver biopsy performed 5 days before the liver transplant. $(a, b)$ Liver showing $25 \%$ parenchyma necrosis with bridging necrosis, acidophilic bodies, mixed inflammatory infiltrate and sinusoidal lymphocytosis (a: $\times 100 ;$ b: $\times 400)$. (c) EBV in situ hybridization showing occasional EBV-positive B lymphocytes ( $\times 400$, arrows). (d) Liver function tests before and after diagnosis of the EBV. LT: liver transplant; ACV: acyclovir; ALP: alkaline phosphatase; AST: aspartate aminotransferase; ALT: alanine aminotransferase.

phoproliferative disease.

\section{Discussion}

We describe a case of ALF-associated with EBV infection in a previously healthy elderly woman that underwent successful emergent liver transplantation.

ALF, also termed "fulminant liver failure", is a sudden and severe hepatic injury in patients without previous liver diseases. The clinical manifestation usually includes loss of hepatic function, coagulopathy, hepatic encephalopathy and in many cases, progressive multi-organ failure [1,2]. Despite recent advances in intensive care management, mortality rates remain high [6]. The only therapy of proven benefit is emergent liver transplantation [7]. It has been reported that 5-year survival rate is about $80 \%$ in patients who underwent emergency liver transplantation for ALF [8].

The etiologies of ALF vary greatly by country and have evolved over time. In the Unites States, drug-induced ALF, mainly acetaminophen, accounts for about $58 \%$ of cases, and hepatitis A and B accounts for $14 \%$ of cases according to the US Acute Liver Failure Study Group data [3]. Thirteen percent of cases are of indeterminate etiology. These cases often follow a relatively insidious presentation before patients quickly develop liver failure, and rates of survival are poor without transplantation. Proposed potential causes include novel viruses and exposure to toxin. Recent studies showed that the cause may be related to EBV infection [9]. In our case, the cause of ALF was initially thought to be diclofenac, because the patient had been taking diclofenac for about 5 months prior to admission, and other causes such as acetaminophen, infectious agents and metabolic diseases were excluded through serological tests; however, the biopsy and blood EBV DNA PCR results confirmed that EBV is the major cause of ALF, and diclofenac may have played no or a minor role in this process.

EBV-associated ALF is very rare, accounting for only 0.21 $\%$ of adult ALF cases. It usually occurs in adults younger than 40 years of age, which is consistent with the fact that young adult patients tend to have symptomatic infectious mononucleosis $[10,11]$. It has been reported that patients with neoplasm, CMV and HIV and patients on immunosuppressant drugs are at high risk for EBV-associated ALF, although recent studies also showed that immunocompetent patients can also develop ALF, indicating that other unknown risk factors may also be involved in the development of EBV-associated ALF [12-14]. In adult aged 60 years or older, there were only two cases of EBV-associated ALF in the literature; however, both of them had impaired immune system: one had just started steroids and the other had recent heart surgery [15-17]. In our case, the patient does not have any of the above mentioned problems and did not take any immunosuppressant medications. Her immune system appears normal although her lymphocyte counts were low. This is the first case of EBV reactivation-associated ALF in a healthy elderly patient. This indicates that in older immunocompetent patients presenting with ALF, a primary EBV infection or EBV reactivation should be considered when 
other causes have been excluded.

Early diagnosis of EBV-associated hepatitis was difficult as reliable diagnostic criteria are not clearly defined [11]. Molecular approaches, such as EBV-DNA PCR or EBER-RISH, are frequently used to diagnose EBV-related hepatitis $[9,18]$. Serological EBV tests such as VCA-IgM, VCA-IgG, EA-D and EBNA can also help to detect a recent infection or a prior infection, or a reactivated EBV infection, although they are not reliable, especially in the elderly, as they have significantly higher titers of the serum tests than young adults $[9,18]$. In our case, the liver biopsy 1 week before admission showed 25\% hepatic necrosis, some atypical B lymphocytes with positive EBV RNA. In explanted liver, there was over $75 \%$ necrosis, significantly increased atypical B cells and strongly positive expression of EBV RNA, which is consistent with the progression of the liver failure. We also tested the blood EBV level by PCR 4 days after liver transplantation and it was extremely high, indicating a severe active EBV infection. Steroids started after liver transplantation may accelerate the rapid replication. The EBV serology tests 10 days after liver transplantation showed very high VCA-IgG but normal VCA-IgM, indicating that this is an EBV reactivation. Moreover, the liver enzyme showed cholestatic enzyme changes after transplantation with continuously increased ALP. Interestingly, ALP rapidly decreased after we started acyclovir. We think that the increased ALP was due to damage caused by EBV infection to the implanted liver. Treatment with acyclovir should be strongly considered after liver transplantation for ALF from EBV to prevent infection of the liver graft during the period of high level immunosuppression.

There are some limitations in our case. First, we were not able to diagnose EBV hepatitis until after evaluation of the explanted liver. Liver transplantation could be potentially avoided if an early diagnosis of EBV hepatitis could have been made. Second, the EBV PCR test was done after transplantation, when patient had already been on steroids. The extremely high EBV PCR value may not reflect the severity of EBV infection, as steroids can suppress the immune system and EBV could replicate faster than expected. Third, the serological test was done 10 days after we started acyclovir, which may not show the initial changes of EBV infection.

In addition, the use of diclofenac in this patient since a few months ago might have caused some initial injury to the liver. Diclofenac is reported to cause liver injuries by two main mechanisms, hypersensitivity and metabolic aberration [19, 20]. Acute liver injury caused by diclofenac is thought to be due to hypersensitivity and usually presents within days to a few weeks after starting diclofenac, which can result in fatal liver failure. Metabolic aberration can cause persistent mild liver injury and once diclofenac is stopped, the liver can recover quickly [20]. In our patient, diclofenac might have caused mild liver injury initially; however, the reactivation of EBV plays a major role in developing ALF. We are confident that EBV is the cause of ALF in the patient based on liver enzyme changes, characteristic histopathological changes, and most importantly strong EBV RNA expression in the explanted liver.

In conclusion, our case demonstrates that EBV infection or reactivation may be under-recognized as a cause of ALF. Early recognition and diagnosis is extremely important, how- ever difficult. In both healthy young and elderly adults with ALF, EBV should be considered when other causes are excluded. Liver biopsy should be strongly considered in patients with unexplained ALF with hepatitis pattern and EBV RNA by in situ hybridization should be mandatory if sinusoidal lymphocytosis is present. Emergent liver transplantation is the only definitive treatment option, with very favorable prognosis for such patients. Treatment with acyclovir should be strongly considered after liver transplantation for ALF from EBV to prevent infection of the liver graft during the period of high level immunosuppression.

\section{References}

1. Bernal W, Auzinger G, Dhawan A, Wendon J. Acute liver failure. Lancet. 2010;376(9736):190-201.

2. Bernal W, Wendon J. Acute liver failure. N Engl J Med. 2013;369(26):2525-2534.

3. Ostapowicz G, Fontana RJ, Schiodt FV, Larson A, Davern TJ, Han SH, McCashland TM, et al. Results of a prospective study of acute liver failure at 17 tertiary care centers in the United States. Ann Intern Med. 2002;137(12):947954.

4. Kofteridis DP, Koulentaki M, Valachis A, Christofaki M, Mazokopakis E, Papazoglou G, Samonis G. Epstein Barr virus hepatitis. Eur J Intern Med. 2011;22(1):73-76.

5. Nakazawa A, Nakano N, Fukuda A, Sakamoto S, Imadome K, Kudo T, Matsuoka K, et al. Use of serial assessment of disease severity and liver biopsy for indication for liver transplantation in pediatric Epstein-Barr virus-induced fulminant hepatic failure. Liver Transpl. 2015;21(3):362-368.

6. Bernal W, Hyyrylainen A, Gera A, Audimoolam VK, McPhail MJ, Auzinger G, Rela M, et al. Lessons from look-back in acute liver failure? A single centre experience of 3300 patients. J Hepatol. 2013;59(1):74-80.

7. Lee WM, Squires RH, Jr., Nyberg SL, Doo E, Hoofnagle JH. Acute liver failure: Summary of a workshop. Hepatology. 2008;47(4):1401-1415.

8. Chan G, Taqi A, Marotta P, Levstik M, McAlister V, Wall W, Quan D. Long-term outcomes of emergency liver transplantation for acute liver failure. Liver Transpl. 2009;15(12):1696-1702.

9. Drebber U, Kasper HU, Krupacz J, Haferkamp K, Kern MA, Steffen HM, Quasdorff M, et al. The role of Epstein-Barr virus in acute and chronic hepatitis. J Hepatol. 2006;44(5):879-885.

10. Feranchak AP, Tyson RW, Narkewicz MR, Karrer FM, Sokol RJ. Fulminant Epstein-Barr viral hepatitis: orthotopic liver transplantation and review of the literature. Liver Transpl Surg. 1998;4(6):469-476.

11. Mellinger JL, Rossaro L, Naugler WE, Nadig SN, Appelman H, Lee WM, Fontana RJ. Epstein-Barr virus (EBV) related acute liver failure: a case series from the US Acute Liver Failure Study Group. Dig Dis Sci. 2014;59(7):16301637.

12. Hadinoto V, Shapiro M, Greenough TC, Sullivan JL, Luzuriaga K, Thorley-Lawson DA. On the dynamics of 
acute EBV infection and the pathogenesis of infectious mononucleosis. Blood. 2008;111(3):1420-1427.

13. Sitki-Green D, Covington M, Raab-Traub N. Compartmentalization and transmission of multiple epsteinbarr virus strains in asymptomatic carriers. J Virol. 2003;77(3):1840-1847.

14. Losavio AD, Te HS. Epstein-barr virus: an unusual cause of cholestatic hepatitis in older adults. Gastroenterol Hepatol (N Y). 2007;3(2):101-105.

15. Papatheodoridis GV, Delladetsima JK, Kavallierou L, Kapranos N, Tassopoulos NC. Fulminant hepatitis due to Epstein-Barr virus infection. J Hepatol. 1995;23(3):348350.

16. Cacopardo B, Nunnari G, Mughini MT, Tosto S, Benanti F, Nigro L. Fatal hepatitis during Epstein-Barr virus reactivation. Eur Rev Med Pharmacol Sci. 2003;7(4):107-
109.

17. Pelletier LL, Borel DM, Romig DA, Liu C. Disseminated intravascular coagulation and hepatic necrosis. Complications of infectious mononucleosis. JAMA. 1976;235(11):1144-1146.

18. Middeldorp JM, Brink AA, van den Brule AJ, Meijer CJ. Pathogenic roles for Epstein-Barr virus (EBV) gene products in EBV-associated proliferative disorders. Crit Rev Oncol Hematol. 2003;45(1):1-36.

19. O'Connor N, Dargan PI, Jones AL. Hepatocellular damage from non-steroidal anti-inflammatory drugs. QJM. 2003;96(11):787-791.

20. Banks AT, Zimmerman HJ, Ishak KG, Harter JG. Diclofenac-associated hepatotoxicity: analysis of 180 cases reported to the Food and Drug Administration as adverse reactions. Hepatology. 1995;22(3):820-827. 